\title{
Asterosaponin 1 induces endoplasmic reticulum stress-associated apoptosis in A549 human lung cancer cells
}

\author{
YACHAO ZHAO $^{1 *}$, CHANGLIANG ZHU $^{1 *}$, XIAOFEI LI ${ }^{1}$, ZHIPEI ZHANG $^{1}$, YUAN YUAN $^{2}$, \\ YUNFENG NI ${ }^{1}$, TONGGANG LIU ${ }^{1}$, SANMING DENG ${ }^{1}$, JINBO ZHAO ${ }^{1}$ and YUNJIE WANG ${ }^{1}$ \\ ${ }^{1}$ Department of Thoracic Surgery, Tangdu Hospital, Fourth Military Medical University; ${ }^{2}$ Department \\ of Radiology, Xijing Hospital, Fourth Military Medical University, Xi'an 710038, P.R. China
}

Received May 9, 2011; Accepted June 6, 2011

DOI: $10.3892 /$ or.2011.1358

\begin{abstract}
Asterosaponin 1, a new cytostatic agent from starfish, possesses several bioactivities including an antitumor effect. The aim of this study was to investigate the potential anti-proliferative and pro-apoptotic activity of asterosaponin 1 in A549 human lung cancer cells, as well as the potential mechanisms. The results showed that asterosaponin 1 inhibited the proliferation of A549 cells in a dose-dependent manner, and the cytostatic activity resulted from the induction of apoptotic cell death. Asterosaponin 1 increased ER dilation and cytosolic $\mathrm{Ca}^{2+}$ concentration, and enhanced the expression of the ER molecular chaperones GRP78 and GRP94 in a doseand time-dependent manner. In addition, asterosaponin 1 treated A549 cells exerted increased expression and activity of CHOP, caspase-4 and JNK, three essential ER-associated apoptotic molecules. In summary, these results demonstrated that asterosaponin 1 inhibits the proliferation of A549 cells through induction of ER stress-associated apoptosis, making asterosaponin 1 a candidate new anticancer drug for lung cancer therapy.
\end{abstract}

\section{Introduction}

Lung cancer is one of the most common cancers in the world, and is considered as the leading cause of cancer-related death (1). Non-small cell lung cancer (NSCLC), a main type of lung cancer based on histology, accounts for more than $80 \%$ of lung cancers and has a poor prognosis for decades due to its high metastatic potential and resistance to current therapies $(2,3)$. Despite recent advances in treatment strategies, there are no effective clinical therapies for NSCLC patients except for surgery and radiotherapy with several side effects (2). Thus,

Correspondence to: Professor Yunjie Wang, Department of Thoracic Surgery, Tangdu Hospital, Fourth Military Medical University, Xi'an, Shaanxi Province 710038, P.R. China

E-mail: tangduxiongwai@163.com

*Contributed equally

Key words: asterosaponin 1, apoptosis, endoplasmic reticulum stress, A549 cells finding more effective and less harmful anticancer drugs is urgently required to improve clinical outcomes and extend survival for NSCLC patients.

Asterosaponin is a new class of steroidal glycosides extracted and isolated from starfish and responsible for their general toxicity (4). Diverse pharmacological effects induced by asterosaponin and its analogues have been reported, including antibacterial, antiviral, antitumor and antifungal activities (5-7). A previous study has demonstrated that asterosaponin 1 whose chemical structure is shown in Fig. 1, is capable of inhibiting growth and inducing apoptosis in human glioblastoma U87MG cells (8). Novaeguinoside II, one of the newly found asterosaponins, also has been shown to induce apoptosis in U87MG cells by a mitochondrial apoptotic pathway (9). However, the role of asterosaponin 1 in the process of apoptosis in lung cancer cells has not previously been determined. In the current study we therefore, examined the anti-proliferative and pro-apoptotic efficacy of asterosaponin 1 in human lung cancer A549 cells.

The endoplasmic reticulum (ER) is an extensive membranous labyrinth network extending from the nuclear membrane throughout the cytoplasm (10). It functions as a site for protein folding, export and secretion, a pool of free calcium, and a compartment for lipid and sterol synthesis (11). Under certain conditions, such as hypoxia, nutrient deprivation, oxidative stress, DNA damage, or treatment with several pharmacological agents, unfolded and misfolded proteins can accumulate in the ER lumen and result in so-called ER stress $(12,13)$. If the stress is so severe that it cannot be overcome by the protective signal transduction pathways in ER, cells will undergo apoptotic death. ER stress is considered to be associated with a wide range of diseases including cancer, and several pharmacological agents have been found to exert anticancer effects through ER stress-induced apoptosis (14-16). In the present study, we aimed to investigate the molecular mechanism underlying the potential anticancer function of asterosaponin 1 by focusing on the ER stress pathway in human lung cancer A549 cells.

\section{Materials and methods}

Cell culture and treatment. Human lung cancer A549 cells were cultured in Dulbecco's modified Eagle's medium (DMEM) supplemented with $10 \%$ fetal bovine serum and 


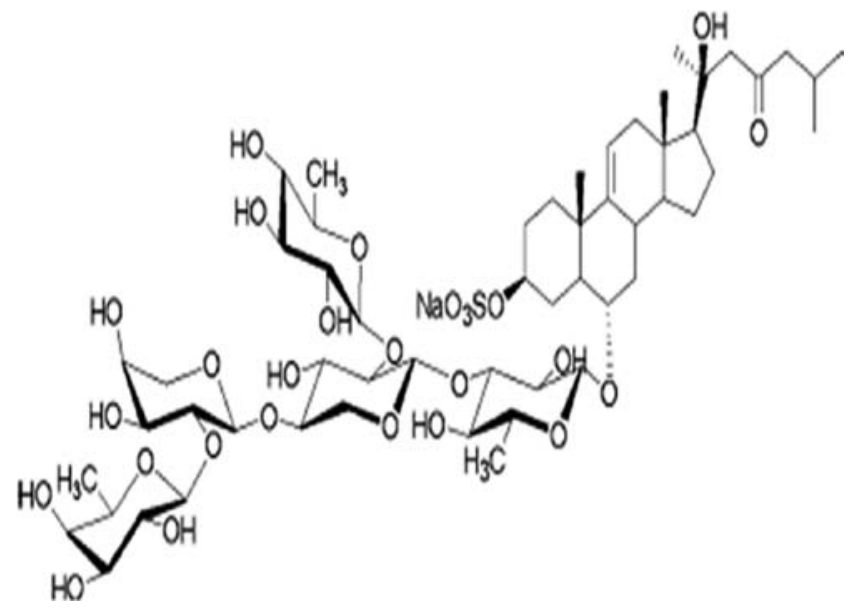

Figure 1. Structure of asterosaponin 1.

$80 \mathrm{U} / \mathrm{ml}$ penicillin/streptomycin. The cells were maintained at $37^{\circ} \mathrm{C}$ in a humidified atmosphere of $95 \%$ air and $5 \%$ $\mathrm{CO}_{2}$, and the culture medium was changed every other day. Asterosaponin 1 was dissolved in $0.1 \%$ dimethyl sulfoxide (DMSO), which had no effect on cell viability, and different concentrations of the sample $(2.5,5$ and $10 \mu \mathrm{g} / \mathrm{ml})$ were added into the culture medium $24 \mathrm{~h}$ before performing the cell viability and apoptosis assays.

MTT assay. Cell viability was measured using the 3-(4,5-dimethylthiazol-2-yl)-2,5-diphenyl tetrazolium bromide (MTT) assay, which assessed the mitochondrial activity by measuring the ability of cells to convert MTT to the blue formazan dye. Briefly, A549 cells were seeded in 96-well plates and subjected to various treatments as described earlier. The MTT solution ( $5 \mathrm{mg} / \mathrm{ml}$ ) was added to each culture well, and the cells were incubated for $4 \mathrm{~h}$ at $37^{\circ} \mathrm{C}$. After the medium was carefully removed, the blue-colored formazan product was dissolved with DMSO and the absorbance was subsequently measured at $570 \mathrm{~nm}$ using a microplate reader. Cell survival rates were expressed as percentage of the value of cells without any treatment.

TUNEL staining. Apoptotic cell death in A549 cells after asterosaponin 1 treatment was detected by the terminal deoxynucleotidyl transferase-mediated dUTP biotin nick end labeling (TUNEL) assay, a method to observe DNA strand breaks in nuclei. For TUNEL staining, cells were seeded on $1.5-\mathrm{cm}$ glass cover slides and treated with different concentrations of asterosaponin 1 for $24 \mathrm{~h}$, after which cells were fixed by immersing slides in freshly prepared $4 \%$ methanol-free formaldehyde solution in PBS for $20 \mathrm{~min}$ at room temperature and permeabilized with $0.2 \%$ Triton X-100 for 5 min. Cells were labeled with fluorescein TUNEL reagent mixture for $60 \mathrm{~min}$ at $37^{\circ} \mathrm{C}$ according to the manufacturer's suggested protocol (Roche, Mannheim, Germany). After that, the number of TUNEL-positive cells was counted by using fluorescence microscopy and the apoptotic rate was determined.

Transmission electron microscopy. Transmission electron microscopy was performed on A549 cells to evaluate the

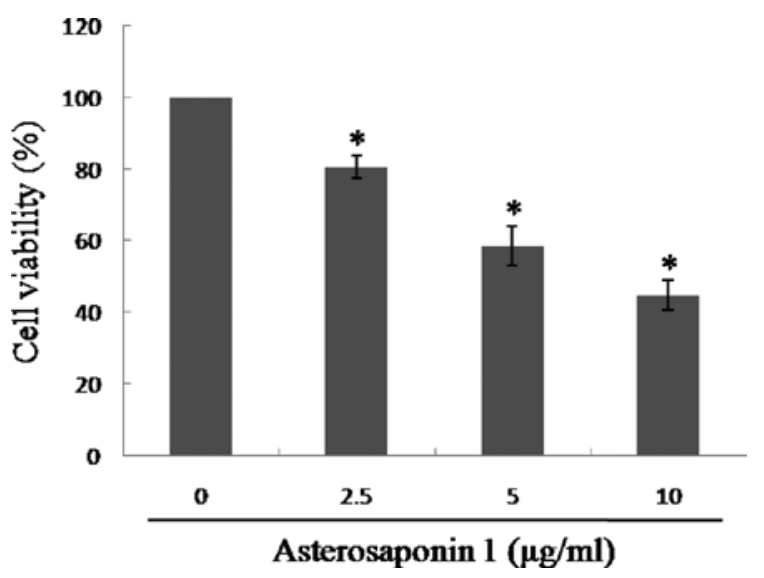

Figure 2. Asterosaponin 1 inhibits the proliferation of A549 cells. After exposure to different concentrations $(2.5,5$ and $10 \mu \mathrm{g} / \mathrm{ml})$ of asterosaponin 1 for $24 \mathrm{~h}$, the cell viability of A549 cells was measured by the MTT assay. The data are presented as the means \pm SD from five independent experiments. ${ }^{*} \mathrm{p}<0.05$ vs. control group.

effects of asterosaponin 1 on ER morphology. Transmission electron microscopy of cells was performed as previously described (17). Briefly, after treatment with $10 \mu \mathrm{g} / \mathrm{ml}$ for $24 \mathrm{~h}$, cells were collected by trypsinization, pelleted by centrifugation, and fixed with $2.5 \%$ glutaraldehyde. The pellets were post-fixed in osmium tetroxide, dehydrated with graded acetone series and finally embedded in Epon 812. Ultrathin sections were cut on an ultramicrotome, stained with uranyl acetate and lead citrate, and examined with a transmission electron microscope.

Measurement of intracellular $\mathrm{Ca}^{2+}$ levels. Free cytosolic calcium was measured using the cell permeant $\mathrm{Ca}^{2+}$-sensitive fluorescent dye Fluo-3AM. After treatment with asterosaponin 1 for $24 \mathrm{~h}$, the medium was removed and replaced with Fluo-3AM at a final concentration of $1 \mu \mathrm{mol} / 1$ for $30 \mathrm{~min}$ at $37^{\circ} \mathrm{C}$. Cells were then washed twice with PBS and determination of Fluo-3 fluorescence was done by flow cytometry. The results were represented as relative fluorescence intensities normalized to untreated controls.

Western blot analysis. Equivalent amounts of protein $(60 \mu \mathrm{g}$ per lane) were loaded and separated by $12 \%$ SDS-PAGE gels, and transferred to polyvinylidene difluoride (PVDF) membranes. Membranes were blocked with 5\% non-fat milk solution in Tris-buffered saline with $0.1 \%$ Triton X-100 (TBST) for $1 \mathrm{~h}$, and then incubated overnight at $4^{\circ} \mathrm{C}$ with the following primary antibody dilutions in TBST: anti-GRP78, GRP94, CHOP, caspase-4, cleaved caspase-4, JNK, p-JNK and $\beta$-actin (1:800). The membranes were then washed and incubated with secondary antibody for $1 \mathrm{~h}$ at room temperature. The blots were developed using chemiluminescence, and the intensities of the bands were quantified with Sigma Gel image analysis software.

Data analysis. Statistical analysis was performed using SPSS 16.0, a statistical software package. All data are presented as mean \pm SD. Statistical evaluation of the data was performed by ANOVA. A value of $\mathrm{p}<0.05$ was considered statistically significant. 


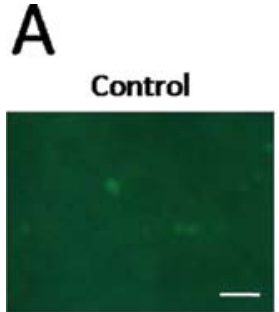

$5 \mu \mathrm{g} / \mathrm{ml}$

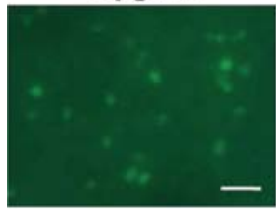

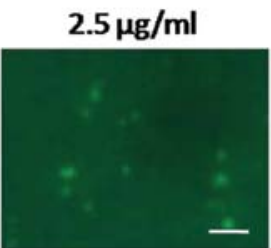

$10 \mu \mathrm{g} / \mathrm{ml}$

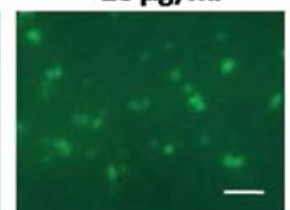

B

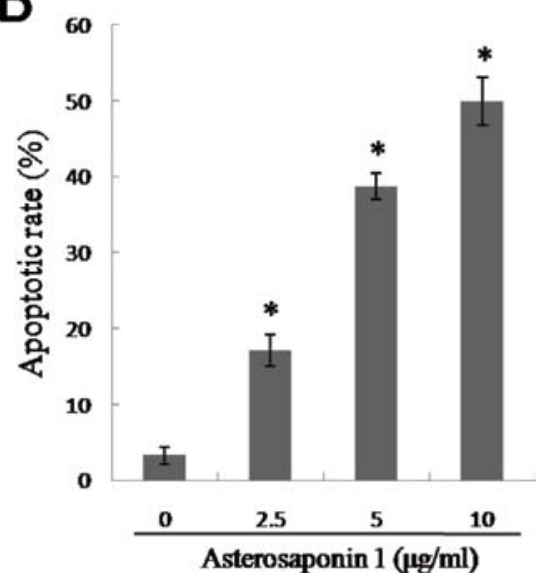

Figure 3. Asterosaponin 1 induces apoptosis in A549 cells. (A) A549 cells were exposed to asterosaponin 1 at different concentrations (2.5, 5 and $10 \mu$ g/ml) for $24 \mathrm{~h}$, stained with TUNEL reagent, and visualized under fluorescence microscopy. (B) The apoptotic rate was estimated by scoring apoptotic cells as indicated in (A). Scale bar, $50 \mu \mathrm{m}$. The data are presented as means \pm SD from six independent experiments. ${ }^{*}$ p $<0.05$ vs. the control group.

A

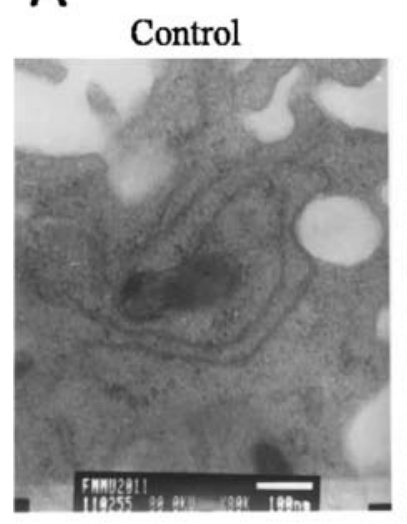

Asterosaponin 1

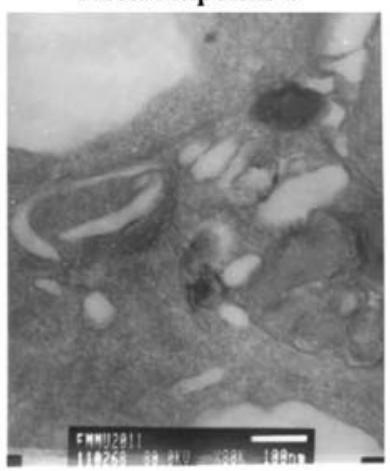

B

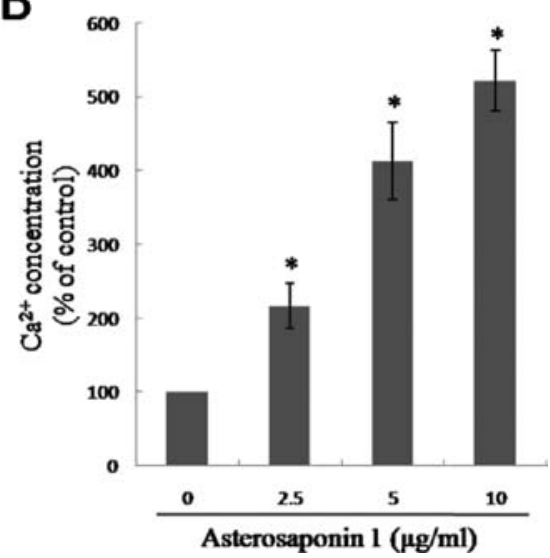

Figure 4. Asterosaponin 1 increases ER dilation and cytosolic $\mathrm{Ca}^{2+}$ concentrations in A549 cells. (A) A549 cells were treated with or without $10 \mu \mathrm{g} / \mathrm{ml}$ asterosaponin 1 for $24 \mathrm{~h}$, and the ER morphology was visualized by transmission electron microscopy. (B) After treatment with asterosaponin 1 at different concentrations $(2.5,5$ and $10 \mu \mathrm{g} / \mathrm{ml})$ for $24 \mathrm{~h}$, A549 cells were stained with Fluo-3AM, and the concentrations of cytosolic Ca ${ }^{2+}$ were calculated by the green fluorescence excited at $488 \mathrm{~nm}$. The data are presented as means \pm SD from five independent experiments. ${ }^{*}$ p $<0.05$ vs. the control group.

\section{Results}

Asterosaponin 1 inhibits the proliferation of A549 cells by inducing apoptosis. To investigate the potential antiproliferative effects of asterosaponin 1 in human lung cancer A549 cells, different concentrations $(2.5,5$ and $10 \mu \mathrm{g} / \mathrm{ml})$ of asterosaponin 1 were added into the culture medium, and the cell viability was measured by the MTT assay $24 \mathrm{~h}$ later. As shown in Fig. 2, asterosaponin 1 decreased the viability of A549 cells in a dose-dependent manner. Next, we investigated the effect of asterosaponin 1-induced apoptosis by using the TUNEL assay (Fig. 3). Compared with the control group, the TUNEL-positive A549 cells significantly increased with the concentrations of asterosaponin 1 added (from 3.3 \pm 1.1 to $17.1 \pm 2.1,38.8 \pm 1.7$ and $49.0 \pm 3.2 \%)$.

Asterosaponin 1 induces ER stress in A549 cells. Transmission electron microscopy was performed on A549 cells to evaluate the effects of asterosaponin 1 on ER morphology (Fig. 4A).
The results showed that asterosaponin 1 increased ER dilation at $10 \mu \mathrm{g} / \mathrm{ml}$ for $24 \mathrm{~h}$, and some vacuoles formed from the destruction of ER structural integrity were observed in asterosaponin 1 treated A549 cells. We further assessed the effect of asterosaponin 1 on the mobilization of $\mathrm{Ca}^{2+}$, and the results of Fluo-3AM staining indicated that asterosaponin 1 increased the cytosolic $\mathrm{Ca}^{2+}$ concentration in a dose-dependent manner in A549 cells (Fig. 4B). Next, we examined the expression of the ER molecular chaperones GRP78 and GRP94 by immunoblotting analyses. As shown in Fig. 5, asterosaponin 1 markedly increased the expression levels of GRP78 in a dose and time-dependent manner, but GRP94 levels were not affected.

Role of ER stress in asterosaponin 1-induced apoptosis of A549 cells. To further shed light on the relationship between asterosaponin 1-induced apoptosis and ER stress, Western blot analysis was used to measure three essential ER-associated apoptotic molecules, including CHOP, caspase-4 and JNK 

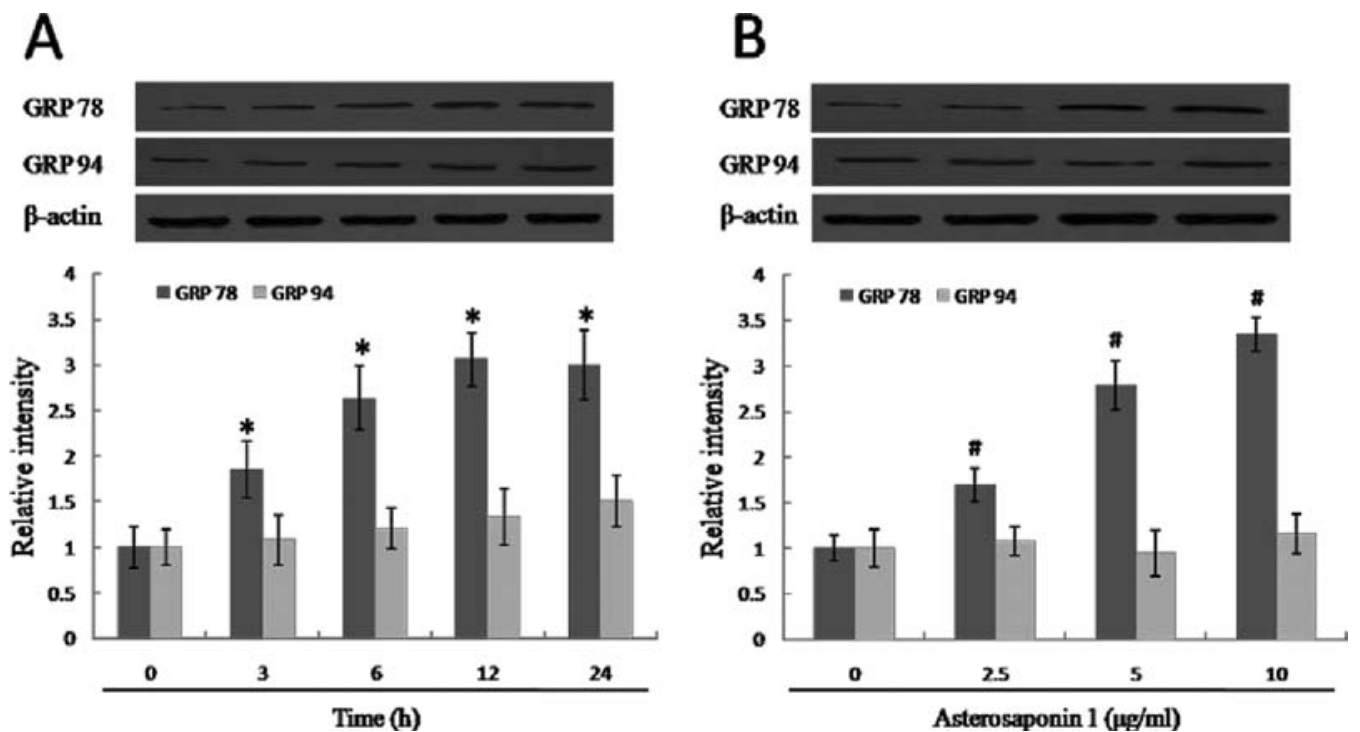

Figure 5. Effects of asterosaponin 1 on ER molecular chaperones. A549 cells were treated with asterosaponin 1 at $10 \mu \mathrm{g} / \mathrm{ml}$ for different time intervals (A), or with different concentrations of asterosaponin 1 for $24 \mathrm{~h}$ (B), and then the GRP78 and GRP94 expressions were examined by Western blot analysis. The data are presented as the means \pm SD from five independent experiments. " $\mathrm{p}<0.05$ and ${ }^{\#} \mathrm{p}<0.05$ vs. the control group.

A

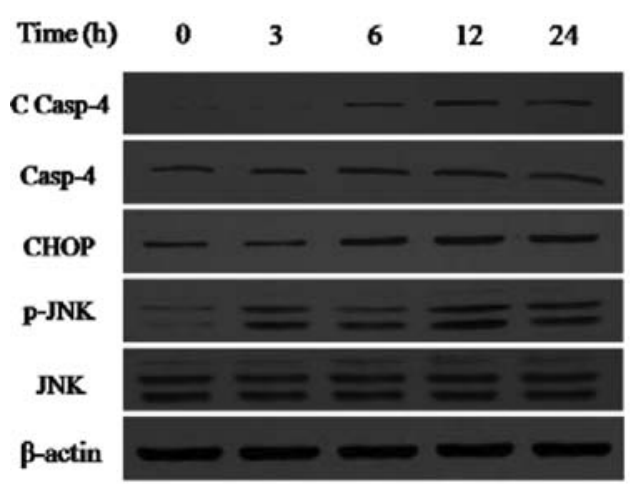

B

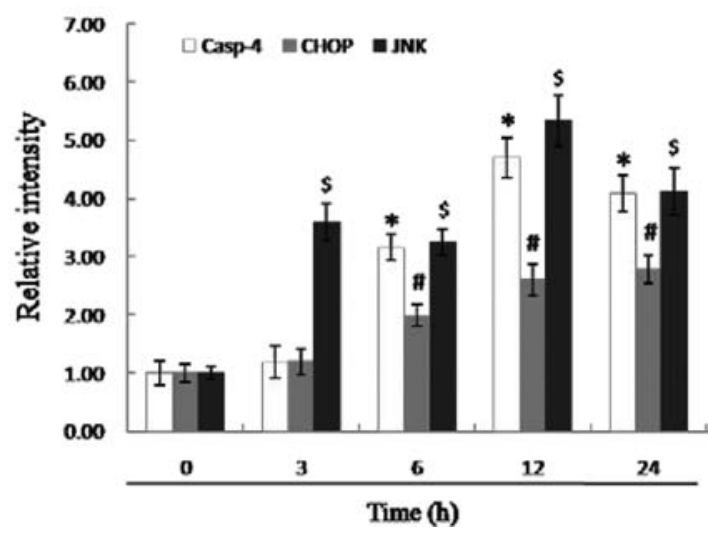

Figure 6. ER stress is involved in asterosaponin 1-induced apoptosis in A549 cells. A549 cells were treated with asterosaponin 1 at $10 \mu \mathrm{g} / \mathrm{ml}$ for different time intervals, and then the activation of caspase-4, CHOP and JNK were detected by Western blot analysis. The data are presented as the means \pm SD from five independent experiments. ${ }^{*} \mathrm{p}<0.05,{ }^{\#} \mathrm{p}<0.05$ and ${ }^{\$} \mathrm{p}<0.05$ vs. the control group.

in A549 cells $24 \mathrm{~h}$ after asterosaponin 1 treatment (Fig. 6). After exposure to $10 \mu \mathrm{g} / \mathrm{ml}$ asterosaponin 1, the expression of CHOP and cleaved caspase- 4 significantly increased from 6 to $24 \mathrm{~h}$ compared to the control group. The expression levels of p-JNK started to increase notably at $3 \mathrm{~h}$ and sustained high expression of p-JNK was observed from 3 to $24 \mathrm{~h}$ after asterosaponin 1 treatment. Furthermore, the total protein levels of caspase-4 and JNK were not markedly affected.

\section{Discussion}

Apoptosis, also known as type I programmed cell death, is an important form of cell death for the survival of multicellular organisms and occurs under several physiological and pathological conditions, such as development or the prevention of oncogenic transformation $(18,19)$. Cancer cells are characterized by their ability to develop diverse anti-apoptotic mechanisms to avoid being killed by apoptosis, and are therefore, resistant to chemotherapy (20). To discover agents capable of activating apoptosis pathways directly and selectively in cancer cells is an excellent therapeutic strategy for many malignancies, including lung cancer (21). In the present study, we found that asterosaponin 1, a new cytostatic agent from the starfish, inhibited the proliferation of human lung cancer A549 cells in a dose-dependent manner, and the cytostatic activity resulted from the induction of cell apoptosis.

Apoptosis can be initiated by several distinct, but interconnected, molecular signaling pathways, among which the extrinsic (mediated by the activation of death receptors) and the intrinsic (mitochondria-dependent signaling) pathways represent the two major well-studied apoptotic processes (22). In the past few decades, ER stress, a more recently discovered 
biochemical cascade, are considered as significant regulators of apoptosis and have drawn much attention. The ER stress is a cellular response to a number of biochemical, physiological to pathological stimuli and can trigger various cellular dysfunctions including accumulation of misfolded proteins and alteration of $\mathrm{Ca}^{2+}$ homeostasis (23). The close relationship between elevated ER stress and apoptotic cell death has been confirmed in many cell types including macrophages, pancreatic $\beta$-cells, neurons and endothelial cells with implication for several human diseases, including cancer $(24,25)$. Our study revealed that with the treatment of asterosaponin 1, the ER of A549 cells dilated and the cytosolic-calcium levels, the reflection of depletion of luminal ER calcium, markedly increased in a dose-dependent manner. These data suggested that ER stress is possibly induced by asterosaponin 1 in human lung cancer A549 cells.

The glucose-related proteins (GRPs) are referred to as ER molecular chaperones because of their ubiquitous expression in endoplasmic reticulum and their ability to assist in protein folding and assembly (11). GRP78 (also known as BiP) and GRP94, two well-characterized members of GRPs, are homologous to cytosolic heat shock protein (HSP) 70 and 90, and are generally considered as molecular markers of ER stress $(26,27)$. Their expression levels are up-regulated in response to ER stress conditions and they are considered as a part of the cellular defense mechanisms to block further cell injury and death (28). In this study, we found that asterosaponin 1 significantly increased the expression of GRP78 in a dose and time-dependent manner, but with no effects on the expression of GRP94. It was reported that honokiol increased the expression of GRP78 but not GRP94 in human chondrosarcoma cells (29), which is similar to our finding in A549 cells, but another experiment in human gastric cancer cells indicated that honokiol induced GRP94 but not GRP78 cleavage (30). Transfection with targeting GRP78 siRNA resulted in up-regulation of GRP94 in HeLa cells, suggesting the compensational feature of GRP94 in the absence of GRP78 (31). All these data suggest that the modulation of GRP78 and GRP94 in ER stress-associated apoptosis was quite complex and might be dependent on cell types. Furthermore, with its calcium binding activity, GRP78 can preserve intracellular $\mathrm{Ca}^{2+}$ homeostasis by regulating the depletion of ER intracisternal $\mathrm{Ca}^{2+}$. Therefore, the up-regulation of GRP78, as well as the increased cytosolic-calcium levels as shown in Fig. 4, are both reflections of ER stress in A549 cells after asterosaponin 1 treatment.

Under the conditions of prolonged and/or severe ER stress, the cell activates several intracellular signaling pathways that lead to apoptotic cell death. The induction of $\mathrm{CHOP}$ and activation of JNK kinase and caspase- 4 are apparently key pro-apoptotic factors that are closely associated with ER stress. CHOP (also known as GADD153), a transcription factor downstream of the PERK-ATF4 axis, was thought to play important roles in the process of ER stress-associated apoptosis via suppressing the transcription of anti-apoptotic Bcl-2 and inducing ROS production (16). The protective effect of CHOP deletion was confirmed in cytokine-induced cell death (32) and in $\mathrm{CHOP}^{-/}$animals against renal damage caused by the ER stress-inducing toxin tunicamycin (33). Caspase-4, a protein predominantly localized in the ER, is specifically cleaved by ER stress but not by other non-ER stimuli, and several studies have identified caspase-4 as a key player in the ER stress-mediated pathway of apoptosis in humans or caspase-12 in rodents (34). The activation of caspase- 4 could further activate caspase- 3 and caspase- 9 without involving the mitochondrial cytochrome c pathway (35). Another molecular mechanism associated with ER stress-induced apoptosis involves the activation of the JNK, which is a member of the MAPK famiy and plays a critical role under various stimuli in both the extrinsic and intrinsic apoptotic pathways (36). It has previously been shown that ER stress can directly trigger the JNK cascade through IRE1 activation (37), or activate JNK via IRE1-mediated recruitment of the scaffolding protein TRAF2 to the ER membrane (38). Our present study showed that asterosaponin 1 increased the expression of CHOP and enhanced the activation of caspase- 4 and JNK, suggesting that asterosaponin 1-induced apoptosis in A549 cells was mediated by its actions on ER stress-associated pro-apoptotic factors.

In conclusion, these results demonstrated that asterosaponin 1 inhibited the proliferation of human lung cancer A549 cells in a dose-dependent manner, and the cytotoxicity was attributable to apoptotic cell death. The asterosaponin 1-induced apoptosis was associated with the ER stress-mediated activation of CHOP, caspase- 4 and JNK. These data have provide a possible cellular mechanism underlying the cytotoxic effect of asterosaponin 1 on human lung cancer A549 cells, and will be helpful for evaluating the potency of asterosaponin 1 as a candidate anticancer drug for the treatment of human lung cancer.

\section{References}

1. Zhao J, Kim JE, Reed E and Li QQ: Molecular mechanism of antitumor activity of taxanes in lung cancer (Review). Int J Oncol 27: 247-256, 2005.

2. Burris HA III: Shortcomings of current therapies for non-small cell lung cancer: unmet medical needs. Oncogene 28 (Suppl 1): S4-S13, 2009.

3. Choi YH: Induction of apoptosis by trichostatin A, a histone deacetylase inhibitor, is associated with inhibition of cyclooxygenase-2 activity in human non-small cell lung cancer cells. Int J Oncol 27: 473-479, 2005.

4. Chludil HD, Seldes AM and Maier MS: Antifungal steroidal glycosides from the patagonian starfish anasteriasminuta: structure-activity correlations. J Nat Prod 65: 153-157, 2002.

5. Tang HF, Yi YH, Li L, Sun P, Zhang SQ and Zhao YP: Asterosaponins from the starfish Culcita novaeguineae and their bioactivities. Fitoterapia 77: 28-34, 2006.

6. Tang HF, Yi YH, Li L, Sun P, Zhang SQ and Zhao YP: Three new asterosaponins from the starfish Culcita novaeguineae and their bioactivity. Planta Med 71: 458-463, 2005.

7. Tang HF, Yi YH, Li L, Sun P, Zhang SQ and Zhao YP: Bioactive asterosaponins from the starfish Culcita novaeguineae. J Nat Prod 68: 337-341, 2005.

8. Cheng G, Zhang X, Tang HF, et al: Asterosaponin 1, a cytostatic compound from the starfish Culcita novaeguineae, functions by inducing apoptosis in human glioblastoma U87MG cells. J Neurooncol 79: 235-241, 2006.

9. Zhou J, Cheng G, Tang HF and Zhang X: Novaeguinoside II inhibits cell proliferation and induces apoptosis of human brain glioblastoma U87MG cells through the mitochondrial pathway. Brain Res 1372: 22-28, 2011.

10. Schmitz A and Herzog V: Endoplasmic reticulum-associated degradation: exceptions to the rule. Eur J Cell Biol 83: 501-509, 2004.

11. Gething MJ and Sambrook J: Protein folding in the cell. Nature 355: 33-45, 1992.

12. Mori K: Tripartite management of unfolded proteins in the endoplasmic reticulum. Cell 101: 451-454, 2000. 
13. Wang G, Yang ZQ and Zhang K: Endoplasmic reticulum stress response in cancer: molecular mechanism and therapeutic potential. Am J Transl Res 2: 65-74, 2010.

14. Choi HH, Shin DM, Kang G, et al: Endoplasmic reticulum stress response is involved in Mycobacterium tuberculosis protein ESAT-6-mediated apoptosis. FEBS Lett 584: 2445-2454, 2010.

15. Huang $X$, Zhang Z, Jia L, Zhao Y, Zhang X and Wu K: Endoplasmic reticulum stress contributes to vitamin E succinate-induced apoptosis in human gastric cancer SGC-7901 cells. Cancer Lett 296: 123-131, 2010.

16. McCullough KD, Martindale JL, Klotz LO, Aw TY and Holbrook NJ: Gadd153 sensitizes cells to endoplasmic reticulum stress by down-regulating $\mathrm{Bcl} 2$ and perturbing the cellular redox state. Mol Cell Biol 21: 1249-1259, 2001.

17. Erguven M, Yazihan N, Aktas E, et al: Carvedilol in glioma treatment alone and with imatinib in vitro. Int $\mathrm{J}$ Oncol 36: 857-866, 2010.

18. Portt L, Norman G, Clapp C, Greenwood M and Greenwood MT: Anti-apoptosis and cell survival: a review. Biochim Biophys Acta 1813: 238-259, 2011

19. Vicencio JM, Galluzzi L, Tajeddine N, et al: Senescence, apoptosis or autophagy? When a damaged cell must decide its path - a mini-review. Gerontology 54: 92-99, 2008.

20. Papenfuss K, Cordier SM and Walczak H: Death receptors as targets for anti-cancer therapy. J Cell Mol Med 12: 2566-2585, 2008.

21. Ziedan NI, Kadri H and Westwell AD: The development of proapoptotic cancer therapeutics. Mini Rev Med Chem 8: 711-718, 2008.

22. Garcia-Fuster MJ, Ramos-Miguel A, Rivero G, La Harpe R, Meana JJ and Garcia-Sevilla JA: Regulation of the extrinsic and intrinsic apoptotic pathways in the prefrontal cortex of short- and long-term human opiate abusers. Neuroscience 157: 105-119, 2008

23. Madeo F and Kroemer G: Intricate links between ER stress and apoptosis. Mol Cell 33: 669-670, 2009.

24. Tan Y, Dourdin N, Wu C, De Veyra T, Elce JS and Greer PA: Ubiquitous calpains promote caspase-12 and JNK activation during endoplasmic reticulum stress-induced apoptosis. J Biol Chem 281: 16016-16024, 2006.

25. Yang Q, Kim YS, Lin Y, Lewis J, Neckers L and Liu ZG: Tumour necrosis factor receptor 1 mediates endoplasmic reticulum stress-induced activation of the MAP kinase JNK. EMBO Rep 7: 622-627, 2006 .
26. Lee AS: The glucose-regulated proteins: stress induction and clinical applications. Trends Biochem Sci 26: 504-510, 2001.

27. Um HJ, Bae JH, Park JW, et al: Differential effects of resveratrol and novel resveratrol derivative, HS-1793, on endoplasmic reticulum stress-mediated apoptosis and Akt inactivation. Int J Oncol 36: 1007-1013, 2010.

28. Rao RV, Ellerby HM and Bredesen DE: Coupling endoplasmic reticulum stress to the cell death program. Cell Death Differ 11: 372-380, 2004.

29. Chen YJ, Wu CL, Liu JF, et al: Honokiol induces cell apoptosis in human chondrosarcoma cells through mitochondrial dysfunction and endoplasmic reticulum stress. Cancer Lett 291: 20-30, 2010.

30. Sheu ML, Liu SH and Lan KH: Honokiol induces calpainmediated glucose-regulated protein-94 cleavage and apoptosis in human gastric cancer cells and reduces tumor growth. PLoS One 2: e1096, 2007.

31. Suzuki T, Lu J, Zahed M, Kita K and Suzuki N: Reduction of GRP78 expression with siRNA activates unfolded protein response leading to apoptosis in HeLa cells. Arch Biochem Biophys 468: 1-14, 2007.

32. Cardozo AK, Ortis F, Storling J, et al: Cytokines downregulate the sarcoendoplasmic reticulum pump $\mathrm{Ca}^{2+}$ ATPase $2 \mathrm{~b}$ and deplete endoplasmic reticulum $\mathrm{Ca}^{2+}$, leading to induction of endoplasmic reticulum stress in pancreatic beta-cells. Diabetes 54: 452-461, 2005.

33. Zinszner H, Kuroda M, Wang X, et al: CHOP is implicated in programmed cell death in response to impaired function of the endoplasmic reticulum. Genes Dev 12: 982-995, 1998.

34. Hitomi J, Katayama T, Eguchi Y, et al: Involvement of caspase-4 in endoplasmic reticulum stress-induced apoptosis and Abetainduced cell death. J Cell Biol 165: 347-356, 2004.

35. Yukioka F, Matsuzaki S, Kawamoto K, et al: Presenilin-1 mutation activates the signaling pathway of caspase-4 in endoplasmic reticulum stress-induced apoptosis. Neurochem Int 52: 683-687, 2008.

36. Dhanasekaran DN and Reddy EP: JNK signaling in apoptosis. Oncogene 27: 6245-6251, 2008.

37. Urano F, Wang X, Bertolotti A, et al: Coupling of stress in the ER to activation of JNK protein kinases by transmembrane protein kinase IRE1. Science 287: 664-666, 2000.

38. Nishitoh H, Saitoh M, Mochida Y, et al: ASK1 is essential for JNK/SAPK activation by TRAF2. Mol Cell 2: 389-395, 1998. 